\section{Heated-Tip AFM: Applications in \\ Nanocomposite Polymer Membranes and Energetic Materials}

Jason P. Killgore ${ }^{1}$, William King ${ }^{2}$, Kevin Kjoller ${ }^{3}$ and René M. Overney ${ }^{1}$

1 Dept. of Chem. Eng., Univ. of Washington, Seattle, WA 2 Dept. of Mech. Sci. and Eng, Univ. of Illinois at Urbana-Champaign, IL 3 Anasys Instruments, Santa Barbara, CA kevin@anasysinstruments.com

\section{Introduction:}

Atomic Force Microscopy (AFM) is a key technique for the measurement and analysis of samples when nanoscale topography is of interest. It offers a number of complementary probing modes that extend an AFM's measurement capability to a wide range of material and transport properties of surfaces, including hardness, friction, conductivity and adhesion among others. Sample temperature controlled AFM extends the study of surface morphology and properties to include changes in the material phases.

Recently, silicon microfabricated AFM cantilevers that have integrated heaters, as shown in figure 1, have become commercially available. These cantilevers were initially developed for probe based data storage by researchers at IBM Zurich[1], Figure 1. With the availability of these cantilevers, AFM measurements can be performed where the tip is heated as opposed to the sample. Heated Tip AFM (HT-AFM) refers to AFM operation where a heated tip is used instead of a normal tip to locally heat the sample surface. A number of AFM modes (tapping/contact/force volume etc.) can accommodate a heated tip to yield new information tied to the thermal properties of the sample. Figure 2 shows an example of heated tips to the study of a polystyrene-polypropylene polymer blend. The HT-AFM mode was used to improve the discrimination of the two components in the phase image, which occurs when the tip is heated near the transition temperature of one of the components. The three micrometer scan size images show the high resolution capabilities of the heated tips as well as the ability to locally modify the sample surface.
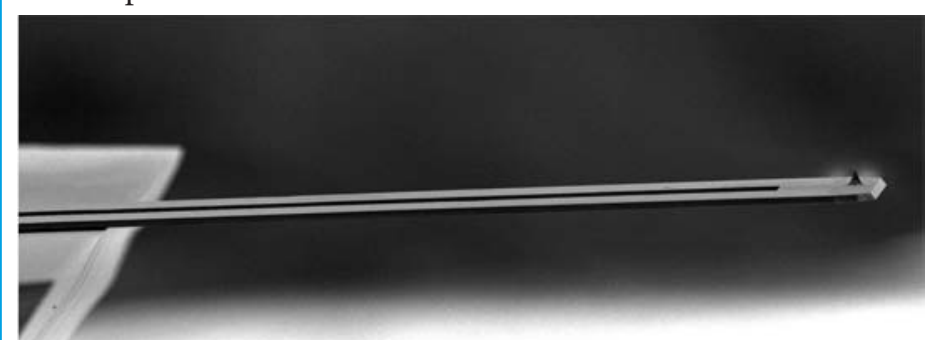

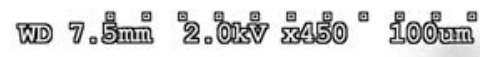

Fig 1: An SEM image of the microfabricated Si cantilever with integrated heater.

Compared to substrate heating methods, HT-AFM offers some distinct advantages. Foremost is the ability to heat to higher temperatures. With substrate heating, a significant temperature rise of the materials in contact with the sample can take place, causing problems with the calibration of the scanner as well as the possibility of damaging the scanner at high temperatures. Another advantage

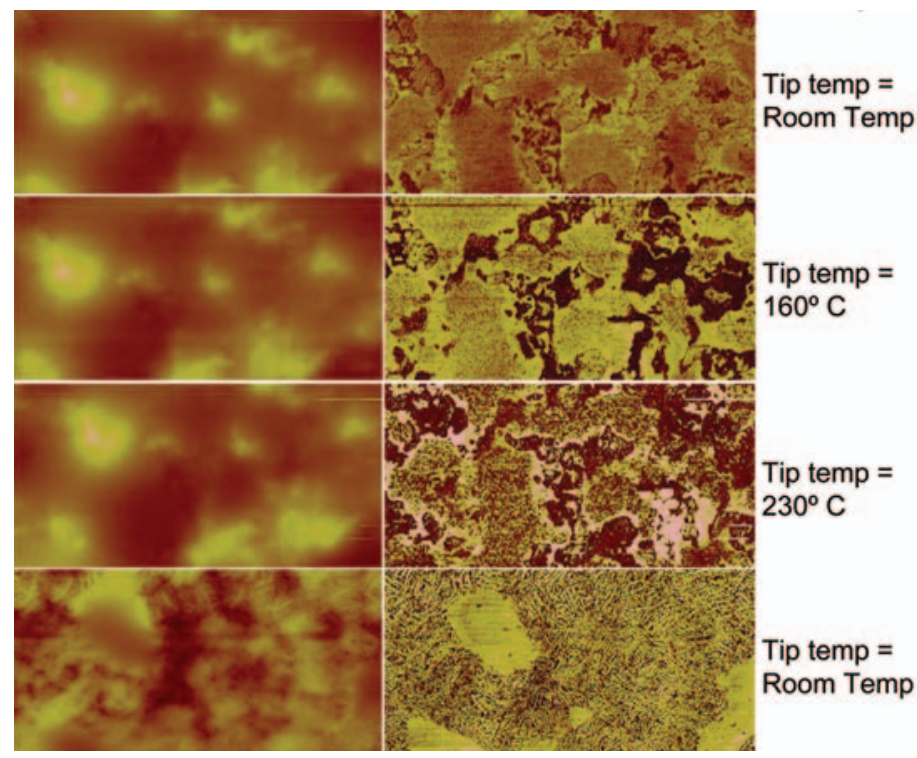

Fig 2: Tapping mode images of a PS/PP Blend with a 3 um scan size. The topography is on the left and the phase on the right.

with the heated tip is that it exhibits much less thermally induced drift. With substrate heating, due to the large area that is being heated, spatial displacements of many microns can be observed over relatively small temperature ranges. As demonstrated in figure 2 of the polymer blend sample, the heated tip method typically shows displacements of less than a micrometer over a temperature range of hundreds of degrees. Other advantages include the ability to operate at very high heating rates due to the low thermal mass, and the ability to perform multiple measurements on specimens that are highly sensitive to thermal history.

A specific technique within the HT-AFM family of techniques is nano Thermal Analysis (nano-TA). This is a local thermal analysis technique that enables one to obtain quantitative information on melt temperature $\left(\mathrm{T}_{\mathrm{m}}\right)$ or glass transition temperatures $\left(\mathrm{T}_{\mathrm{g}}\right)$. Initially the surface can be visualised at nanoscale resolution with the AFM enabling the user to select the spatial locations where they would like to investigate the thermal properties of the surface. The user then obtains the phase transition temperatures by applying heat locally via the probe tip and measuring the thermomechanical response. The nano-TA data displays the cantilever deflection plotted against the tip temperature. This measurement is analogous to the well established technique of thermo-mechanical analysis

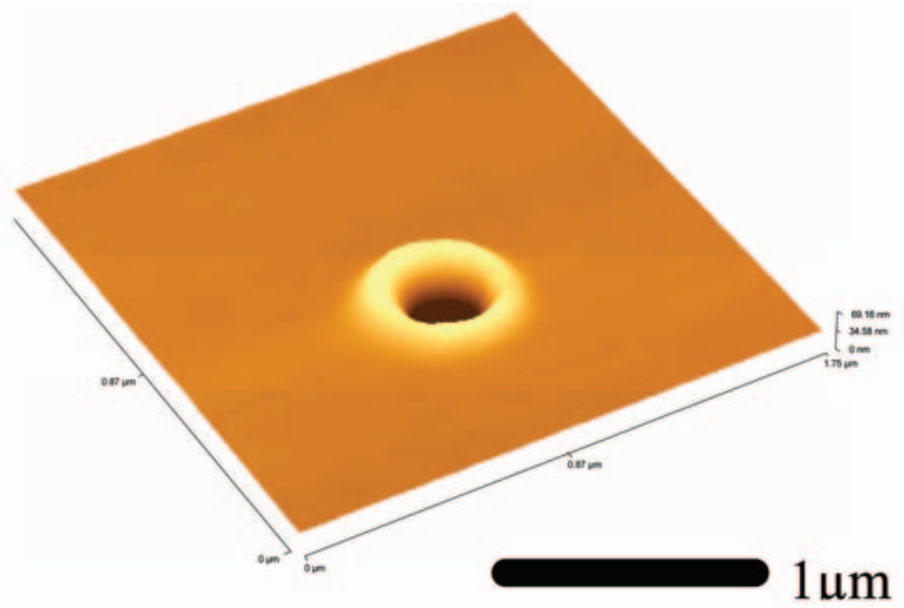

Fig 3: nano-TA on PTMSP. 


\section{GUPRA TM $^{\text {- }}$}

\section{Imaging beyond Expectations}

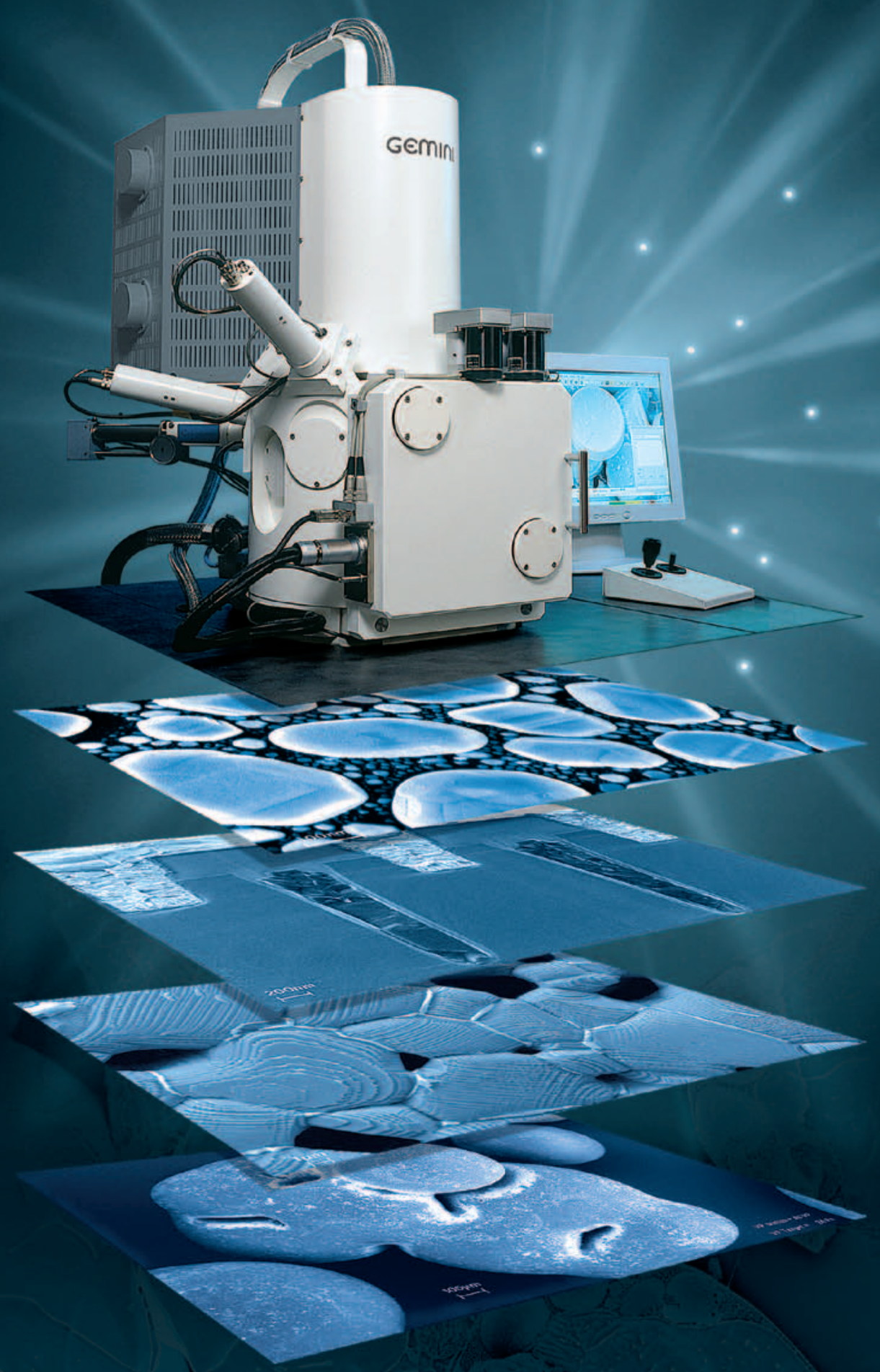

The versatile ultra high resolution FESEM for semiconductor applications, materials analysis, life science and variable pressure solutions.

The new SUPRA ${ }^{\mathrm{TM}}$ combines four instruments in one:

- Ultra high resolution FESEM over the complete voltage range: $1.0 \mathrm{~nm} @ 15 \mathrm{kV}, 1.7 \mathrm{~nm} @ 1 \mathrm{kV}$, $4.0 \mathrm{~nm} @ 0.1 \mathrm{kV}$.

- FESEM for handling large shaped specimens.

- Full analytical FESEM with probe currents up to $3 \mathrm{nA}$ achievable.

- Variable pressure technology to investigate non-conducting specimens without prior preparation.

Carl Zeiss SMT Inc

One Zeiss Drive

Thornwood, New York 10594 USA
Tel. $+1914 / 7477700$

Fax $+1914 / 6817443$

info-usa@smt.zeiss.com

www.smt.zeiss.com/nts

\section{ZDINS}



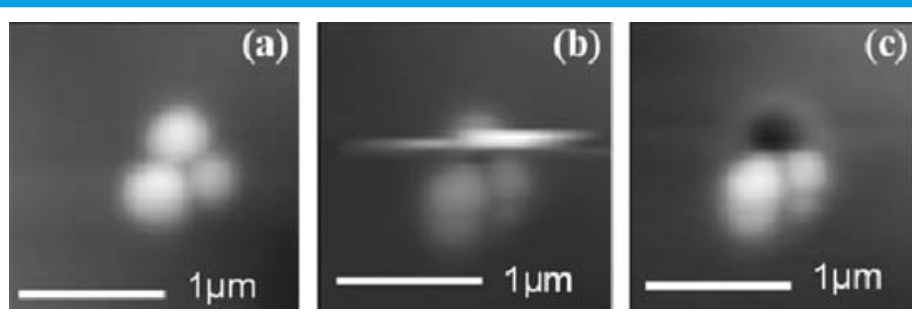

Fig 4: Series of scanning heated tip images taken at tip temperatures at $220^{\circ} \mathrm{C}(\mathrm{a}), 290{ }^{\circ} \mathrm{C}(\mathrm{b})$ and $300^{\circ} \mathrm{C}(\mathrm{c})$. The streaking in image $b$ shows the debonding event and subsequent dragging of the particle.

(TMA). Events such as melting or glass transitions that result in the softening of the material beneath the tip produce a downward deflection of the cantilever.

HT-AFM has been used for a range of applications primarily focused within the polymer and pharmaceutical industries. The measurements that have been demonstrated in the polymer industry include analysis of blend samples, thin films, and nano-scale inclusions. In the pharmaceutical industry, HT-AFM has been used to map crystallinity and polymorphs as well as for the identification of components in compressed tablets and tablet coats. Following are descriptions of two new applications of the HT-AFM mode that broaden the range of measurements possible with this unique capability.

\section{Nanocomposite Polymer Membranes:}

Poly trimethyl silyl propyne (PTMSP) has garnered attention since its discovery due to its very high permeability, high $\mathrm{T}_{\mathrm{g}}$ and exhibited reverse selectivity[2,3]. Recently, it was the discovered that the permeability and reverse selectivity could be significantly enhanced by the addition of silica nanoparticles.[4-8]. While the importance of the nanoscale PTMSP-silica interface has been acknowledged, most of the data currently published is based on larger scale measurements and little is known about the local nanoscale properties of the material. This is primarily due to the challenge of obtaining convenient access to the molecular mobility of material arrangements that are interfacially-constrained. The availability of the thermal probes enables analysis of the interface and characterization of the polymer via the HT-AFM mode.

High purity PTMSP was blended with $200 \mathrm{~nm}$ diameter silica

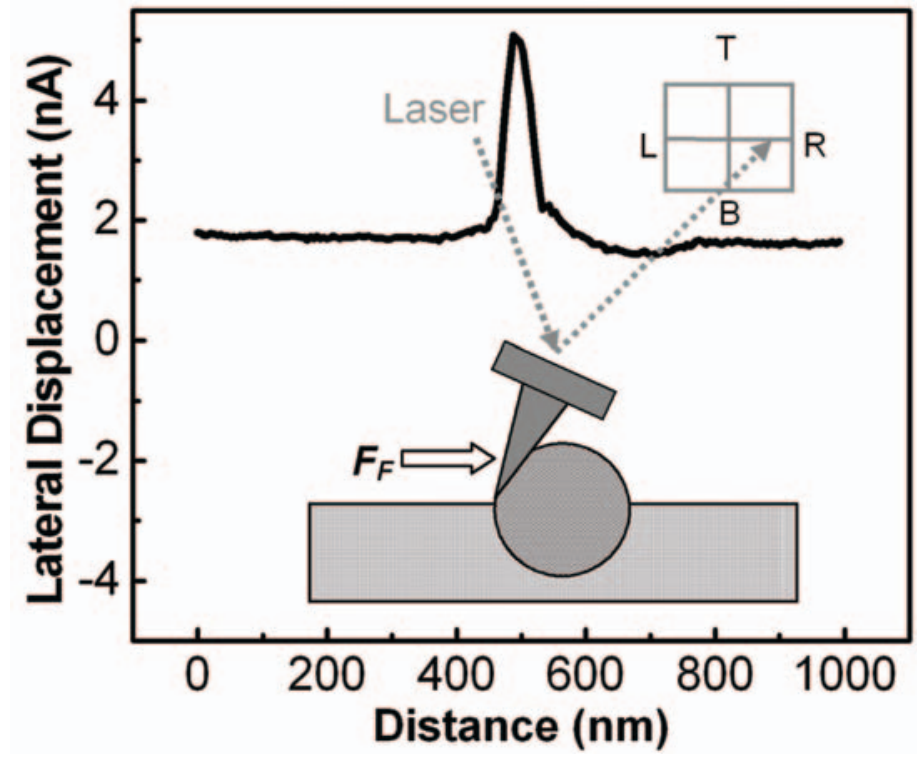

Fig 5: Characteristic lateral force scan across a stable silica particle when scanned with a heated tip. particles. The silica particles were initially prepared in a dilute silica-toluene dispersion. PTMSP was added to the silica-toluene dispersion to make a $3 \mathrm{wt} \%$ solution. The solution was spun cast onto a glass substrate, producing a film thickness of $\sim 1 \mu \mathrm{m}$.

Nano-TA on the PTMSP membrane revealed a $\mathrm{T}_{\mathrm{g}}$ of $190^{\circ} \mathrm{C}$ using a $60^{\circ} \mathrm{C} / \mathrm{min}$ heating rate. Figure 3 is a surface plot image of the thermomechanical analysis indent caused by the temperature ramp. The diameter of the indent created in nano-TA is dependent on a number of factors including heating rate, melt viscosity and when the heated tip is retracted after the transition temperature. If the intent of the experiment is to reduce the affected area of the sample, the tip can be retracted as soon as the transition is reached, which can result in sub $100 \mathrm{~nm}$ indents. The tip can also be kept on the surface after the initial surface transition to probe subsurface components.

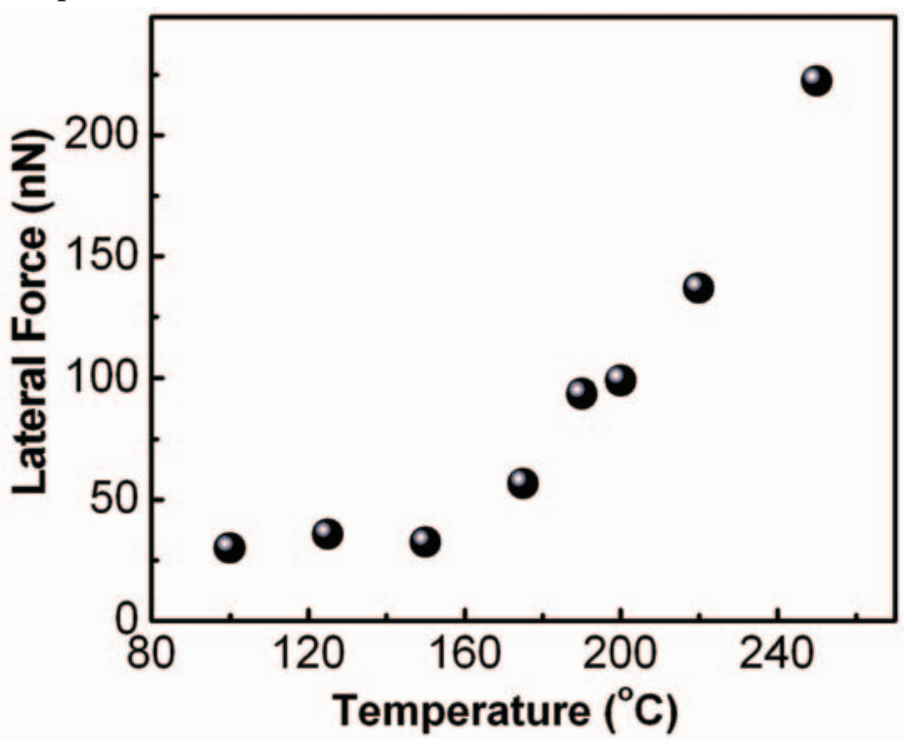

Fig 6: Maximum lateral force subjected to particle as a function of temperature.

When operating the heated tip in scanning mode, the tip is constantly moving, and the sample never reaches thermal equilibrium with the tip. As a result, it is possible to scan with the tip above the $T_{g}$ of the material without initiating a thermal transition at the surface. The actual temperature at the surface depends on

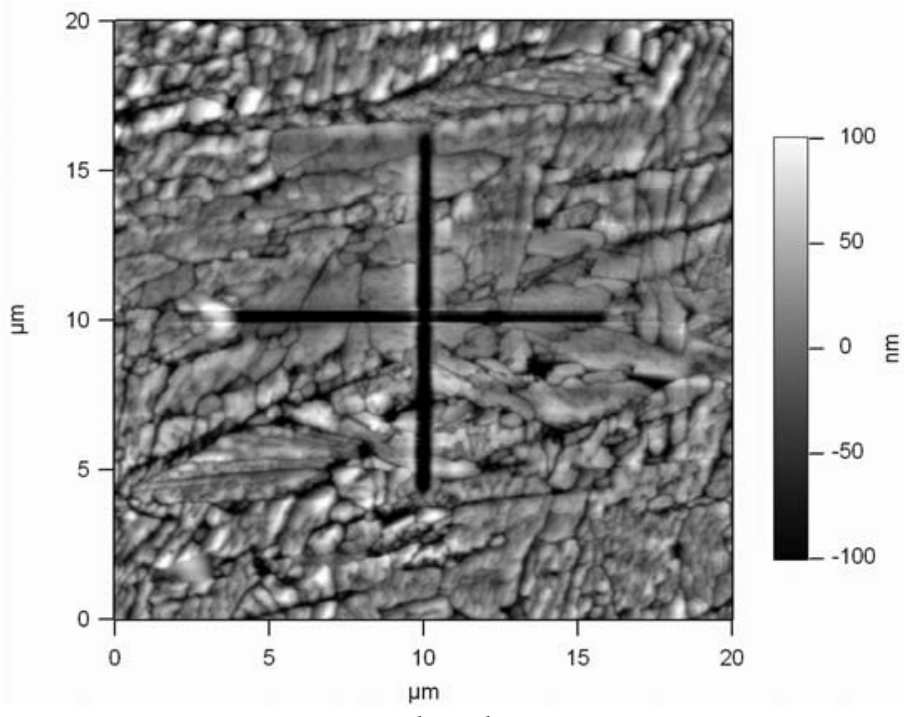

Fig 7: Pattern written using heated tip. 


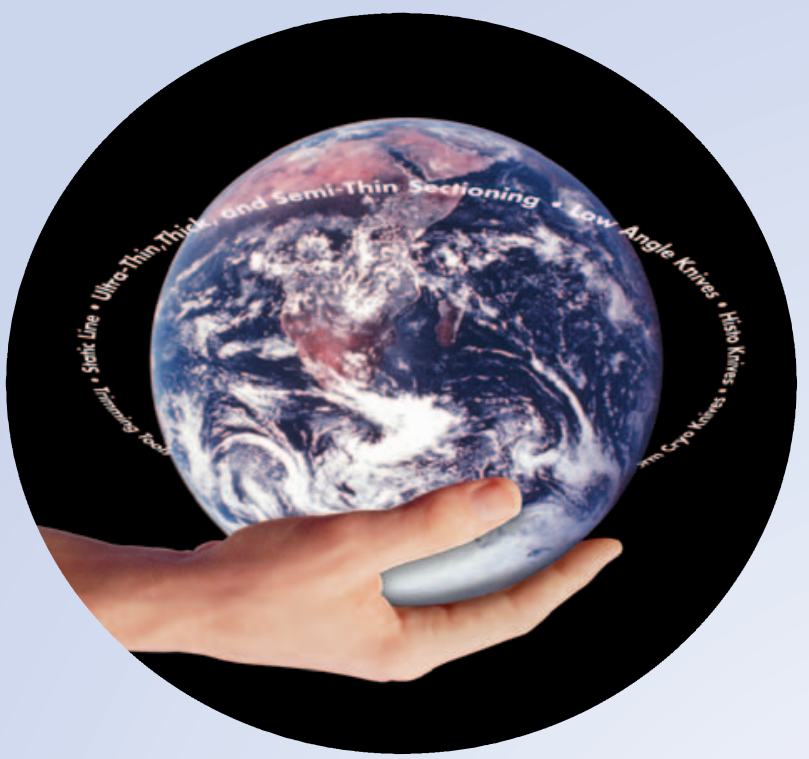

THE WORLD OF MICROSCOPY \& MICROTOMY SOLUTIONS IS WITHIN YOUR REACH WITH
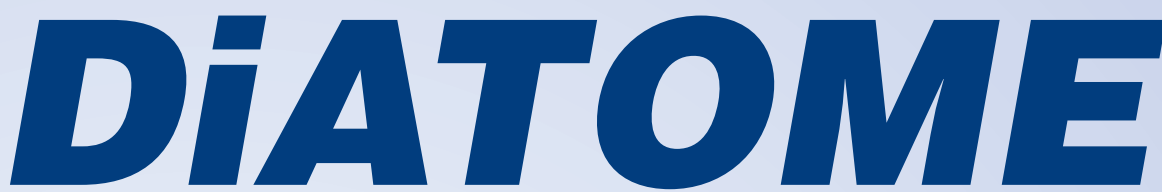

With 36 years of Developing, Innovating \& Discovering under their belts, DiATOME is the only Diamond Knife manufacturer that does not sit around waiting for technology to happen ... they make it happen leaving the other Diamond Knife manufacturers light years behind.

1970 ... DIATOME introduces the FIRST DIAMOND KNIFE.

1980 ... DiATOME introduces the FIRST CRYO DIAMOND KNIFE.

1983 ...DIATOME introduces the FIRST SEMI DIAMOND KNIFE FOR ALTERNATING SECTIONING.

1985 ... DIATOME introduces the FIRST LOW ANGLE $\left(35^{\circ}\right)$ DIAMOND KNIFE.

- 1986 ... DiATOME introduces the FIRST "HISTO" THICK SECTIONING DIAMOND KNIFE.

1989 ... DiATOME introduces the FIRST STATIC ELIMINATOR \& CRYO DIAMOND TRIMMING TOOL. (Revolutionizing Cryo Ultramicrotomy as we know it)

1999 ... DiATOME introduces the FIRST \& ONLY CRYO-P DIAMOND KNIFE. (A Cryo Knife with a Patented Platform for section pick up)

\ 2001 ... DIATOME introduces the CRYO IMMUNO DIAMOND KNIFE.

(The First Cryo Knife with a Diamond Plateau: Optimized pick-up for best section quality in immuno-cytochemistry!)

- 2004 ... DiATOME introduces the REVOLUTIONARY ULTRA SONIC DIAMOND KNIFE.

> 2005 ... DIATOME introduces the CRYO-TRIM $25^{\circ}$ OPTIMIZING TRIMMING WITH DIAMOND BLADES.

$\checkmark 2006$... DIATOME introduces the FIRST DIAMOND KNIVES FOR AFM AT ROOM \& LOW TEMPERATURES.

\ 2006 ... DIATOME introduces the CRYO 25 DIAMOND KNIFE FOR SECTIONING FROZEN HYDRATED SPECIMENS.

\section{DIATOME THANKS THEIR CUSTOMERS FOR 36 YEARS OF PATRONAGE!}
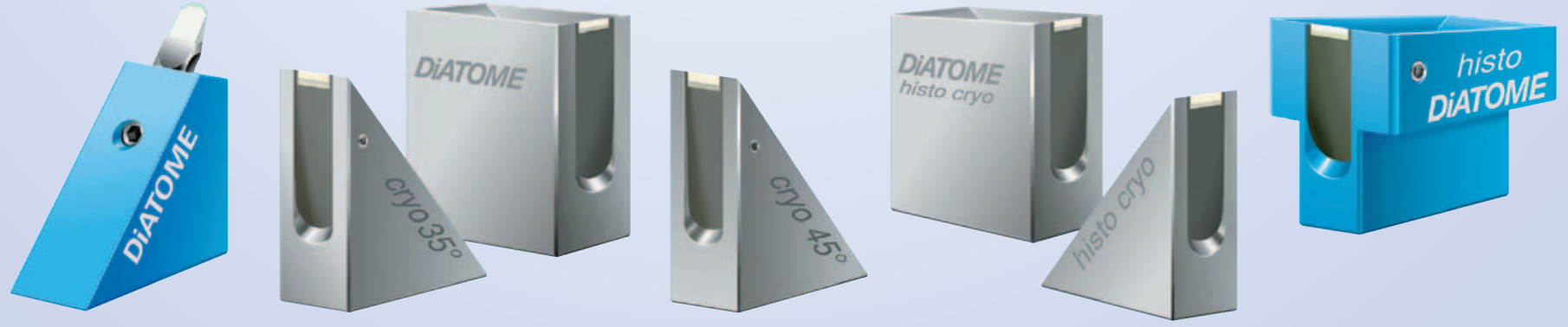

For a copy of our complete product catalog or individual brochure on any of our products, please call or write today, or visit us on the web at: www.emsdiasum.com

DHATOME - 1560 Industry Road P.O. Box 410 - Hatfield, PA 19440 


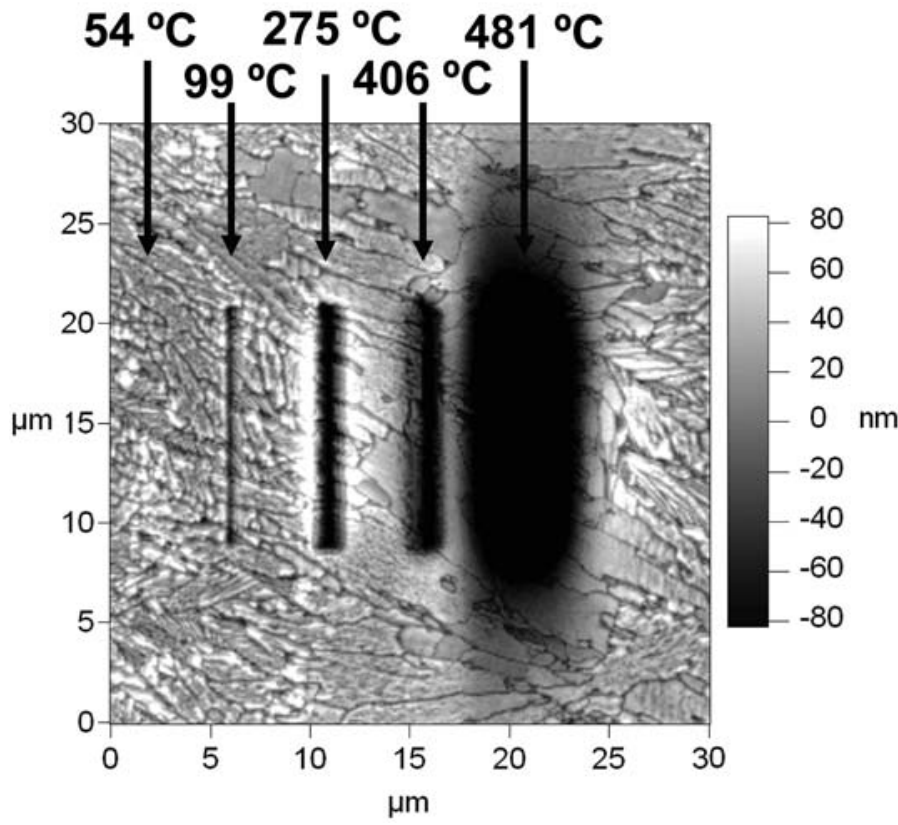

Fig 8: PETN response to different tip temperatures.

the contact resistance, tip temperature, and scan speed. Figure 4 shows a series of heated tip scans on a PTMSP composite where the tip was scanned over exposed silica particles. When a critical temperature is reached $\left(290^{\circ} \mathrm{C}\right.$ for the series under investigation), the particle is debonded from the matrix. The lack of matrix deformation around the particle suggests that the debonding occurred below $\mathrm{T}_{\mathrm{g}}$ and most likely in an elastic manner.

The lateral forces associated with debonding were determined by monitoring the lateral force microscopy signal. Figure 5 shows a characteristic lateral force signal during a single line scan as well as a diagram showing the origin of the signal. The large peak in the lateral force signal is due to the torsional deflection of the cantilever as the tip contacts the particle. In a subsequent experiment, another particle was debonded while the maximum in the lateral force signal was monitored as the tip temperature was increased. As shown in Figure 6, increasing temperature leads to an increasing peak lateral force on the particle. Below the $\mathrm{T}_{\mathrm{g}}$ value of the PTMSP, the lateral force is relatively constant, but increases significantly as the tip temperature is increased above $\mathrm{T}_{\mathrm{g}}$. The maximum observed force at $10^{\circ} \mathrm{C}$ below the debonding temperature was $220 \mathrm{nN}$. The increase in lateral force could be due to a deforming lever or the increase in adhesion, which is typical between the tip and sample above the $\mathrm{T}_{\mathrm{g}}$. These initial debonding experiments illustrate the potential of HT-AFM for adhesive bond strength measurements between embedded inorganic nanoparticles and their organic matrix and may offer a unique way to gain direct insight into interfacial properties of reverse selective membranes.

\section{Energetic Materials:}

Energetic materials exhibit a dramatic release of stored chemical energy as thermal and mechanical energies. The primary difference between an energetic material and any material that undergoes a chemical decomposition process is the rate at which the decomposition occurs. The decomposition rate is determined by a number of factors including the particle characteristics (chemical composition, size, and morphology), the magnitude and duration of the reaction stimulus, and material confinement. Energetic materials often have nanometre-scale polycrystallinity, voids, and
/ or defects, and it is widely believed that nanoscale properties and phenomena within these materials play a key role in their macroscopic behaviour. [9-11] Due to the lack of nanoscale thermal probes previously, these properties have not been extensively studied.

In recent published research, HT-AFM and nano-TA have been used to study local thermal decomposition in an energetic material with a heated tip, and measure the effects of tip temperature on the energetic material response. [12]. A thin film of Pentaerythritol Tetranitrate (PETN) was prepared at a thickness of $\sim 250 \mathrm{~nm}$ on a glass slide.

When the heated AFM cantilever tip was scanned in contact with the energetic material, heating from the tip could induce nanoscale melting and/or decomposition in the energetic material film. Local thermal decomposition with a heated tip provides a unique method of controlling both the size and spatial resolution of voids in the energetic material. Figure 7 shows a simple “+” pattern written in the PETN film, demonstrating the high spatial resolution and registry of the technique. There was no noticeable pileup or residue, indicating that the material was completely decomposed or evaporated during the thermal writing.

Thanks to the ability to control the temperature of the thermal probes over a wide range, the response at various tip temperatures could be determined, as shown in figure 8. In this experiment, the heated tip was scanned along a line while the tip was held at five different temperatures. The lowest temperature tested, $54^{\circ} \mathrm{C}$, produced no change to the PETN. However, at $99^{\circ} \mathrm{C}$ and above, the heated tip was able to write into the PETN. For the areas decomposed at higher temperatures, the PETN crystals near the decomposed area were noticeably larger than in the unmodified sample regions, suggesting that this type of measurement may be 
useful for studying grain coarsening and aging in energetic materials.

The rate of material reacted was studied by scanning the heated tip over a $5 \mu \mathrm{m}$ square on the PETN film. The images shown in Fig. 9 are larger images taken when the tip is at room temperature after the $5 \mu \mathrm{m}$ scan. The smaller scan began at the bottom of the image and moved up. In the upper image, the $5 \mu \mathrm{m}$ took 1290 seconds to complete and 660 seconds for the lower image. In this experiment, much of the PETN that was heated was removed, but unlike the decomposed lines of Figs. 7 and 8, some of the PETN filled in behind. Furthermore, it appears as if the polycrystalline structure of the PETN orients in a columnar fashion from the top to bottom of the image. When heated, the PETN can either go through a phase transition (sublimation or melt/evaporation) into the gas phase or decompose. We hypothesize that the PETN was melted or evaporated at the heated tip, and some subsequently recondensed onto the previously scanned area along the direction of the strongest temperature gradient. Less material condensed within the scanned square for the longer scan and slower tip speed. The longer dwell time of the heated tip may have allowed the melted / evaporated PETN to diffuse farther from the heated source.

\section{Conclusions:}

The above applications of heated tip AFM demonstrate the range of materials that can be analyzed. HT-AFM can be used to not only differentiate between material components or phases within a material. It also allows for the manipulation of the micro/nanostructure of materials that could be used to study diffusion rates and produce controlled nanoscale features. In the nanocomposite field, it can be employed to investigate the lateral forces exerted during particle-matrix debonding and by studying particle matrix adhesion, which consequently could lead to the design of improved interfaces. From testing the nanometer-scale thermo-mechanochemical response of an energetic material to analyzing polymeric nanocomposites, the high resolution imaging capability, wide range of tip temperatures and heating rates offer unique new capabilities for material analyses.

\section{References:}

[1] W.P. King, T.W. Kenny, K.E. Goodson, G.L.W. Cross, M. Despont, U. Dürig, H. Rothuizen, G. Binnig, and P. Vettiger, "Atomic Force Microscope Cantilevers for Combined Thermomechanical Data Writing and Reading," Applied Physics Letters 78, pp. 1300-1302, 2001.

[2] Masuda T, Isobe E, Higashimura T and Takada K 1983 Journal of the American Chemical Society 105 7473-4.

[3] Masuda T, Tang B Z, Tanaka A and Higashimura T 1986 Macromolecules 19 1459-64.

[4] Merkel T C, Freeman B D, Spontak R J, He Z, Pinnau I, Meakin P and Hill A J 2002 Science 296 519-22.

[5] Merkel T C, He Z, Pinnau I, Freeman B D, Meakin P and Hill A J 2003 Macromolecules 36 6844-55.

[6] De Sitter K, Winberg P, D’Haen J, Dotremont C, Leysen R, Martens J A, Mullens S, Maurer F H J and Vankelecom I F J 2006 Journal of Membrane Science 278 83-91.

[7] Prabhakar R S, Merkel T C, Freeman B D, Imizu T and Higuchi A 2005 Macromolecules 38 1899-910.

[8] Andrady A L, Merkel T C and Toy L G 2004 Macromolecules 37 4329-31

[9] Armstrong, R. W. Journal de Physique IV 1995, 5, (c4), 89-102.

[10]Dlott, D. D. Materials Science and Technology 2006, 22, (4), 463-473.

[11] Armstrong, R. W.; Baschung, B.; Booth, D. W.; Samirant, M. Nano Letters 2003, 3, (2), 253-255.

[12] King, W. P.; Saxena, S.; Nelson, B. A; Weeks, B. L.; Pitchimani, R. Nano Letters 2006, 56, 930-933.

\section{Less Hardware. More Image. No Rival.}

\section{The New Dual Standard.}

Our Elion ${ }^{\mathrm{Tw}}$ and $3 \mathrm{Max}^{\mathrm{m}}$ two-in-one detection platforms bring powerful new applications to leading dual beam, FIB and SEM systems.

Elion" ion mode (left) and electron mode (right) @ 50 pA FIB current.

3 Max ${ }^{*}$ enhanced SE3 image (left) and in-lens image (right) with otherwise identical conditions.
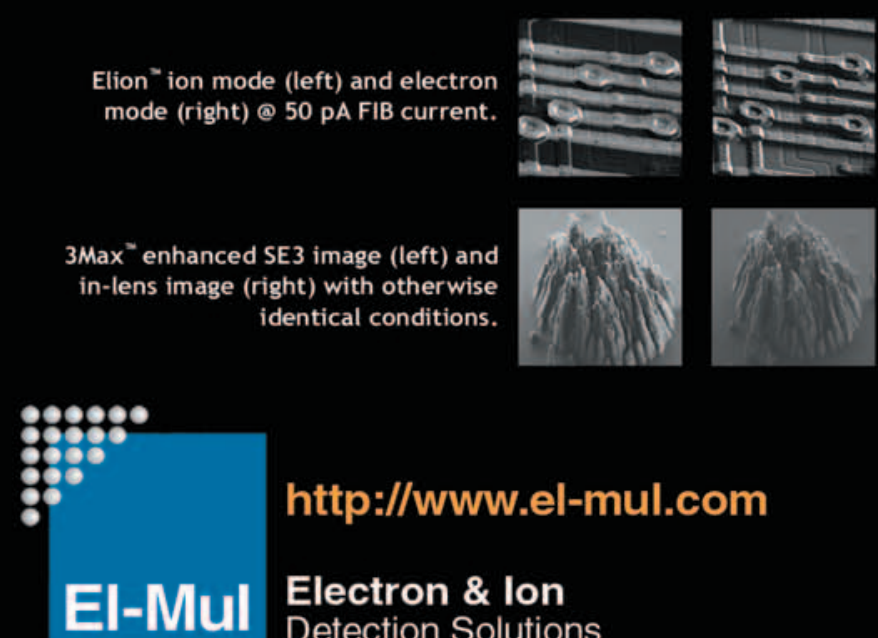

http://www.el-mul.com

Electron \& Ion

Detection Solutions

You can detect the difference. 